\title{
Recursive Least-Squares Adaptive Channel Estimation for Spatial Modulation Systems
}

\author{
Mohammed Mujahid Ulla Faiz, Samir Al-Ghadhban, and Azzedine Zerguine \\ Department of Electrical Engineering \\ King Fahd University of Petroleum \& Minerals \\ Dhahran 31261, Saudi Arabia \\ E-mail: \{mujahid, samir, azzedine\}@kfupm.edu.sa
}

\begin{abstract}
In this paper, a recursive least-squares (RLS) adaptive channel estimation scheme is applied for spatial modulation (SM) system over a block fading multiple-input-multiple-output (MIMO) channel. The performance of spatial modulation with channel estimation is compared to vertical Bell Labs layered space-time (V-BLAST) and maximum ratio combining (MRC) transmission schemes for different pilot rates and a fixed 3$\mathrm{b} / \mathrm{s} / \mathrm{Hz}$ spectral efficiency. Computer simulations carried out demonstrate the superiority of SM over V-BLAST and MRC schemes. In addition, the results in this study show that SM is more robust against channel estimation errors than the other MIMO schemes.
\end{abstract}

Index Terms-Block fading, channel estimation, multipleinput-multiple-output (MIMO), recursive least-squares (RLS), spatial modulation (SM), vertical Bell Labs layered space-time (V-BLAST).

\section{INTRODUCTION}

The necessity for higher data rate and higher spectral efficiency are the key elements that are driving research in future wireless communication systems [1]. Multiple-inputmultiple-output (MIMO) transmission scheme is one solution to achieve this by transmitting multiple data streams from multiple antennas [2]. However, the capacity gain resulting from MIMO transmission is dependent on transmit and receive antenna spacing [3]- [4], transmit antenna synchronization [5]- [6], and the algorithm employed to combat the interchannel interference (ICI) at the receiver. Many ICI reduction algorithms have been reported in the literature and among them is the vertical Bell Labs layered space-time (V-BLAST), which is considered as one of the most promising MIMO detection algorithms. Due to ICI caused by coupling multiple symbols in time and space, the complexity of maximum likelihood (ML) detection increases exponentially with the number of transmit antennas [7]. Avoiding ICI helps in reducing receiver complexity, and achieving better performance gains.

In the spatial modulation (SM) system introduced in [8][9] only one antenna remains active during transmission. Therefore, ICI is completely avoided and inter-antenna synchronization (IAS) is not needed as in the case of V-BLAST, where all antennas are transmitting at the same time. However, the detector in [8] is sub-optimal, and hinders the full performance gains achievable by SM. An optimal detector for spatial modulation system presented in [7], assuming full chan- nel knowledge, achieved performance gains of approximately 1.5-3 dB over other transmission schemes, such as V-BLAST and amplitude/phase modulation (APM) with maximum ratio combining (MRC).

In this work, recursive least-squares adaptive channel estimation for spatial modulation system over a block fading MIMO channel is introduced. From the simulation results it is shown that SM with optimal detection and RLS channel estimation outperforms other transmission schemes, such as V-BLAST and MRC for different pilot rates and a fixed 3$\mathrm{b} / \mathrm{s} / \mathrm{Hz}$ spectral efficiency.

This paper is organized as follows: Section II introduces the basic spatial modulation system model. Section III introduces the optimal detection scheme for spatial modulation system. Section IV introduces the recursive least-squares adaptive channel estimation scheme. Section V presents the simulation results, and Section VI reports some conclusions drawn from this work.

\section{Spatial Modulation System Model}

A basic spatial modulation system model is shown in Fig. 1. It comprises of a SM mapper, $N_{t}$ transmitter antennas, MIMO channel $\mathbf{H}, N_{r}$ receiver antennas and a SM optimal detector.

In spatial modulation system, a block of information bits are mapped into two information carrying units: a symbol that was chosen from a constellation diagram, and a unique transmitter antenna number that was chosen from a set of transmitter antennas, which increases the overall spectral efficiency of the spatial modulation system by the base-two logarithm of the number of transmitter antennas [9]. In general, the number of bits that can be transmitted using spatial modulation system is given as follows [8]:

$$
n=\log _{2}\left(N_{t}\right)+m \text {. }
$$

For MQAM modulation, $m$ is the number of bits/symbol and is given by:

$$
m=\log _{2}(M) .
$$

A random sequence of $b$ independent bits enters into the SM mapper, which groups them into $n$ bits and maps them into a constellation vector $\mathbf{x}=\left[\begin{array}{llll}x_{1} & x_{2} & \cdots & x_{N_{t}}\end{array}\right]^{T}$ of size $N_{t}$. Only one of the $x_{j}$ in $\mathbf{x}$ is nonzero as only one antenna remains active during transmission in spatial modulation, where $j$ is the 
mapped transmitter antenna number, $j \in\left[1: N_{t}\right]$. The symbol $x_{j}$ is transmitted from antenna $j$ over an $N_{r} \times N_{t}$ MIMO channel, $\mathbf{H}$. The received signal is given by:

$$
\mathrm{y}=\sqrt{r \rho} \mathbf{H} \mathbf{x}+\eta,
$$

where $r$ denotes the data rate and is given by:

$$
r=\frac{\text { (block length }- \text { number of pilots) }}{\text { (block length) }}
$$

$\rho$ is the average signal-to-noise ratio (SNR) at each receiver antenna, $\eta$ is the additive white Gaussian noise (AWGN) vector given by:

$$
\eta=\left[\begin{array}{llll}
\eta_{1} & \eta_{2} & \cdots & \eta_{N_{r}}
\end{array}\right]^{T}
$$

$\mathbf{H}$ and $\eta$ have independent and identically distributed (i.i.d.) entries according to $\mathcal{C N}(0,1)$.

As only one antenna remains active during transmission, SM mapper outputs a constellation vector of the following form:

$$
\mathbf{x}_{j q}=\left[\begin{array}{lllllll}
0 & 0 & \cdots & x_{q} & \cdots & 0 & 0
\end{array}\right]^{T},
$$

where $j$ represents the activated transmitter antenna, and $x_{q}$ is the $q^{\text {th }}$ symbol from the $M$-ary constellation. When $x_{q}$ is transmitted from the $j^{\text {th }}$ antenna, then (2) can be written as:

$$
\mathrm{y}=\sqrt{r \rho} h_{j} x_{q}+\eta,
$$

where $h_{j}$ is the $j^{\text {th }}$ column of $\mathbf{H}$.

The number of transmitted information bits, $n$, can be adjusted in two different and independent ways - either by changing the modulation scheme employed and/or changing the number of transmitter antennas [8]. For example, three information bits can be mapped into 4QAM and two transmitter antennas. Alternatively, if the channel and interference environment do not allow the use of 4QAM, the same spectral efficiency can be achieved with BPSK and four transmitter antennas.

Some of the advantages of SM over other transmission schemes like V-BLAST and APM are summarized here:

- Interchannel interference (ICI) is completely avoided.

- Inter-antenna synchronization (IAS) is not needed.

- Provides high spectral efficiency.

- Results in a significant reduction in receiver complexity.

- Does not suffer from the error propagation problem that exists in V-BLAST.

\section{Optimal Detection}

The receiver estimates both the transmitted symbol and the transmitter antenna number and uses these two pieces of information to de-map the block of information bits.

The optimal detector is based on the ML principle [7]:

$$
\begin{aligned}
{\left[\hat{\mathrm{j}}_{\mathrm{ML}}, \hat{q}_{\mathrm{ML}}\right] } & =\arg _{j, q} \max p_{\mathbf{Y}}\left(\mathrm{y} \mid \mathbf{x}_{j q}, \mathbf{H}\right), \\
& =\arg _{j, q} \min \sqrt{\rho}\left\|\mathrm{g}_{j q}\right\|_{\mathrm{F}}^{2}-2 \operatorname{Re}\left\{\mathrm{y}^{H} \mathrm{~g}_{j q}\right\},
\end{aligned}
$$

where $\mathrm{g}_{j q}=\mathrm{h}_{j} x_{q}, 1 \leq j \leq N_{t}, 1 \leq q \leq M$, and $p_{\mathbf{Y}}\left(\mathrm{y} \mid \mathbf{x}_{j q}, \mathbf{H}\right)=\pi^{-N_{r}} \exp \left(-\left\|\mathrm{y}-\sqrt{\rho} \mathbf{H} \mathbf{x}_{j q}\right\|_{\mathrm{F}}^{2}\right)$ is the PDF of $\mathrm{y}$, conditioned on $\mathbf{x}_{j q}$ and $\mathbf{H}$. As seen in (5) both the

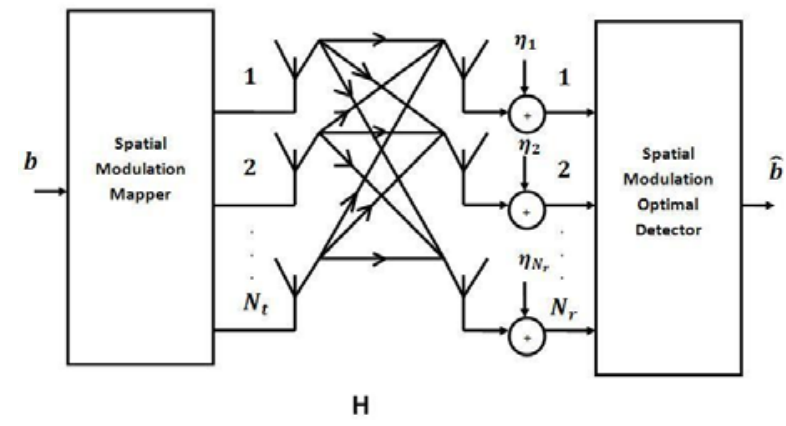

Fig. 1. Spatial modulation system model.

transmitted symbols and the transmitter antenna numbers are detected jointly in optimal detection as opposed to the scheme presented in [8], where the problem is decoupled.

\section{Recursive Least-Squares Adaptive ChanNeL ESTIMATION}

A basic adaptive channel estimation model as shown in Fig. 2 is used here to estimate the MIMO channel $\mathbf{H}$. The same input $\mathbf{u}$ is applied to the adaptive filter and the unknown system. The unknown system output $\mathbf{d}$ is measured in the presence of additive noise $\mathbf{v}$. The output of the adaptive filter $\mathbf{y}$ is subtracted from the output of the unknown system $\mathbf{d}$. The resulting difference is an error signal e, which is used to update the filter coefficients of the adaptive filter. If the system is designed correctly, the adaptive filter's transfer function will converge to the unknown system's transfer function after a known number of iterations. In this paper, the adaptive filter used to estimate the MIMO channel coefficients is implemented using the recursive least-squares (RLS) algorithm.

Given an $N \times 1$ measurement vector $\mathbf{y}$, an $N \times M$ data matrix $\mathbf{H}$ and an $M \times M$ positive-define matrix $\boldsymbol{\Pi}$, the $M \times 1$ solution $\mathbf{w}_{i}$ to the following regularized least-squares problem [10]:

$$
\min _{\mathbf{w}}\left[\mathbf{w}^{*} \mathbf{\Pi} \mathbf{w}+\left\|\mathbf{y}_{i}-\mathbf{H}_{i} \mathbf{w}\right\|^{2}\right]
$$

can be computed recursively as follows. The individual entries of $\mathbf{y}_{i}$ are denoted by $\left\{d_{i}\right\}$, and the individual rows of $\mathbf{H}_{i}$ are denoted by $\left\{\mathbf{u}_{i}\right\}$, i.e.,

$$
\mathbf{y}_{i}=\left[\begin{array}{c}
d_{0} \\
d_{1} \\
d_{2} \\
\vdots \\
d_{i}
\end{array}\right], \quad \mathbf{H}_{i}=\left[\begin{array}{c}
\mathbf{u}_{0} \\
\mathbf{u}_{1} \\
\mathbf{u}_{2} \\
\vdots \\
\mathbf{u}_{i}
\end{array}\right]
$$

Start with $\mathbf{w}_{-1}=0, \mathbf{P}_{-1}=\mathbf{\Pi}^{-1}$ and iterate for $i \geq 0$ :

$$
\begin{aligned}
\gamma_{i} & =\frac{1}{\left(1+\mathbf{u}_{i} \mathbf{P}_{i-1} \mathbf{u}_{i}^{*}\right)} \\
\mathbf{g}_{i} & =\mathbf{P}_{i-1} \mathbf{u}_{i}^{*} \gamma_{i}, \\
\mathbf{w}_{i} & =\mathbf{w}_{i-1}+\mathbf{g}_{i}\left[d_{i}-\mathbf{u}_{i} \mathbf{w}_{i-1}\right] \\
\mathbf{P}_{i} & =\mathbf{P}_{i-1}-\frac{\mathbf{g}_{i} \mathbf{g}_{i}^{*}}{\gamma_{i}}
\end{aligned}
$$




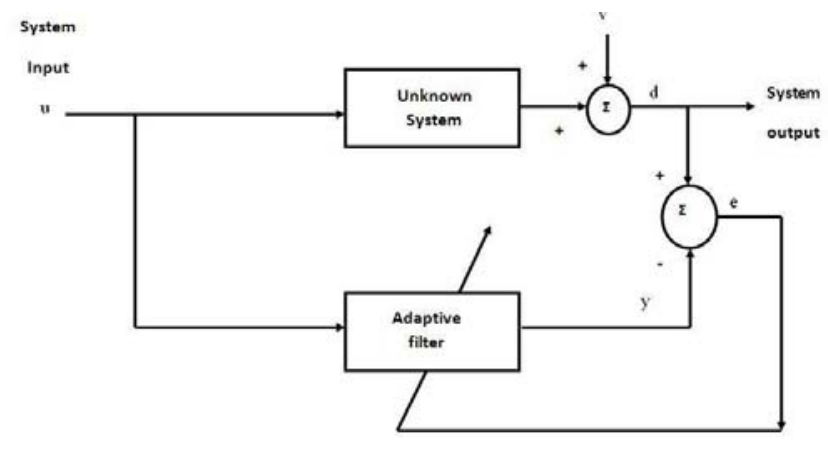

Fig. 2. Adaptive channel estimation model.

At each iteration, the matrix $\mathbf{P}_{i}$ has the following interpretation:

$$
\mathbf{P}_{i}=\left(\boldsymbol{\Pi}+\mathbf{H}_{i}^{*} \mathbf{H}_{i}\right)^{-1}
$$

\section{Simulation Results}

In the simulation, a block Rayleigh fading channel is assumed with AWGN. Monte Carlo simulations are performed for $10^{5}$ channel realizations. The spectral efficiency is fixed at $m=3-\mathrm{b} / \mathrm{s} / \mathrm{Hz}$. The BER performance versus SNR is plotted for $N_{r}=4$ antennas.

For comparison, we use two different transmission setups. The first one is MRC with 8-QAM modulation, and $N_{t}=1$. The second one is V-BLAST with BPSK modulation, $N_{t}=3$, and ordered successive interference cancellation (OSIC) with the minimum mean squared error (MMSE) receiver [11]. SM with BPSK modulation, and $N_{t}=4$ antennas is shown for optimal receiver [7].

Figs. 3, 4, and 5 present the performance of the RLS channel estimator for pilot rates equal to $10 \%, 5 \%$, and $2 \%$ over a block fading channel at $3-\mathrm{b} / \mathrm{s} / \mathrm{Hz}$ spectral efficiency using spatial modulation, V-BLAST and MRC respectively. The results show the expected trade-off between pilot rate and performance. For example, reduction in the pilot rate from $10 \%$ to $5 \%$ results in performance degradation of approximately $2.5 \mathrm{~dB}$ in spatial modulation for BER equal to $10^{-3}$, while the loss is much larger in case of V-BLAST $(\sim 4.5 \mathrm{~dB})$. This is an advantage of SM to be more robust when using practical channel estimation. Also at low pilot rate $(2 \%)$, the system needs much more SNR to perform well.

It is interesting to compare three different MIMO schemes when using practical channel estimation. The result in Fig. 6 shows that SM with RLS channel estimation provides performance improvements of approximately $2.1 \mathrm{~dB}$ over VBLAST and approximately $2.6 \mathrm{~dB}$ over MRC for BER equal to $10^{-3}$ and pilot rate equal to $10 \%$.

In addition, SM with RLS channel estimation is still better than V-BLAST and MRC for pilot rates equal to $5 \%$ and $2 \%$ as shown in Figs. 7-8 respectively.

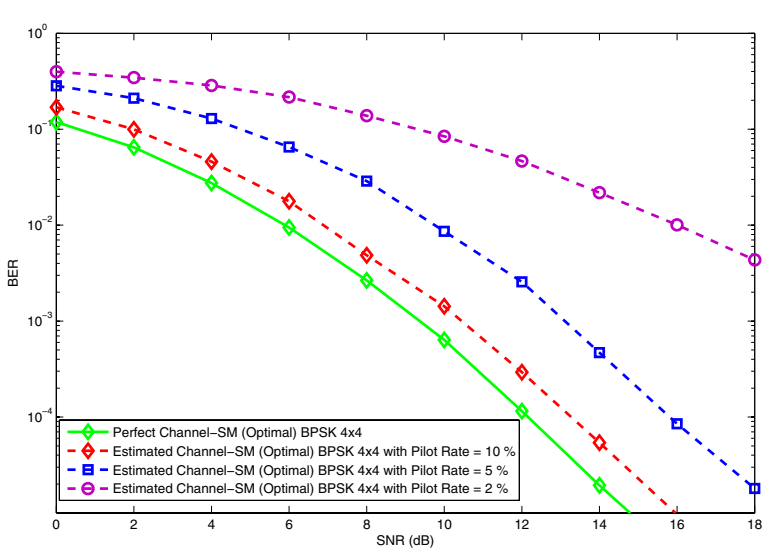

Fig. 3. Performance of the RLS channel estimator for pilot rates $=10 \%, 5 \%$, and $2 \%$ over a block fading channel at $3-\mathrm{b} / \mathrm{s} / \mathrm{Hz}$ using spatial modulation.

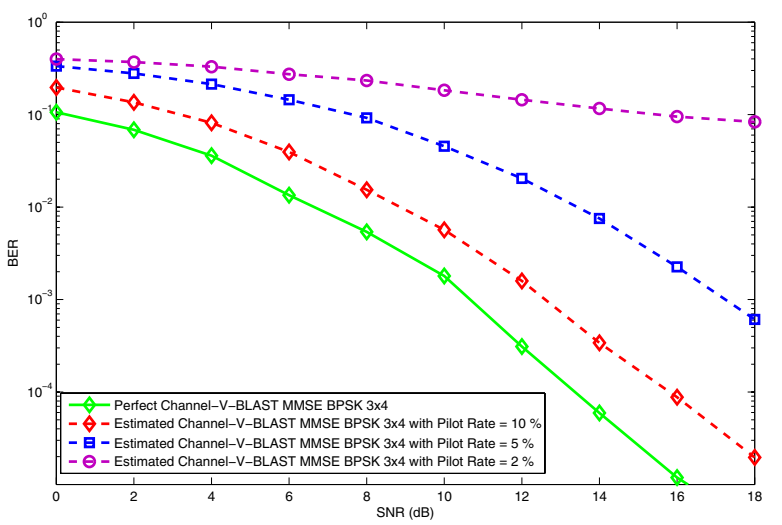

Fig. 4. Performance of the RLS channel estimator for pilot rates $=10 \%, 5 \%$, and $2 \%$ over a block fading channel at $3-\mathrm{b} / \mathrm{s} / \mathrm{Hz}$ using V-BLAST.

\section{CONCLUSIONS}

In this paper, we applied recursive least-squares adaptive channel estimator for spatial modulation system with optimal detection over a block fading MIMO channel. The spectral efficiency is fixed at $3-\mathrm{b} / \mathrm{s} / \mathrm{Hz}$ and pilot rates are varied at $10 \%, 5 \%$, and $2 \%$. The simulation results indicate that SM with optimal detection and RLS channel estimation achieves performance gains of approximately $2.1 \mathrm{~dB}$ over V-BLAST and approximately $2.6 \mathrm{~dB}$ over MRC for BER equal to $10^{-3}$ and pilot rate equal to $10 \%$. In addition, the results show that $\mathrm{SM}$ is more robust with practical channel estimation than V-BLAST.

\section{ACKNOWLEDGMENT}

The authors would like to thank King Fahd University of Petroleum \& minerals for its support. 


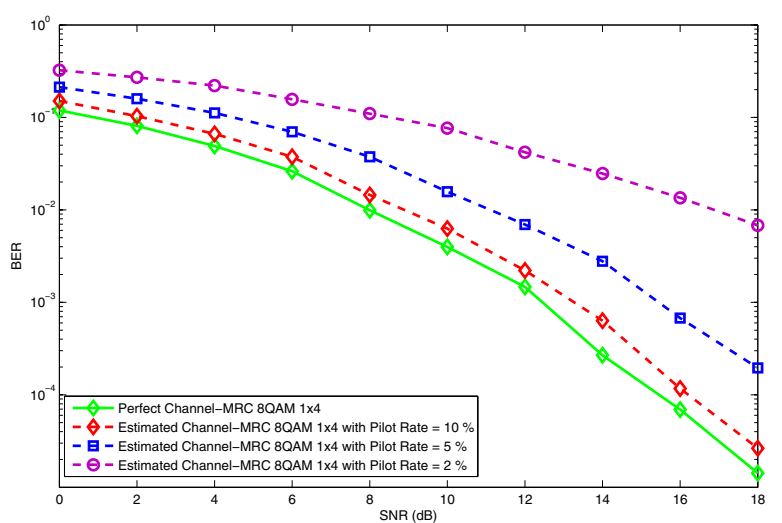

Fig. 5. Performance of the RLS channel estimator for pilot rates $=10 \%, 5 \%$, and $2 \%$ over a block fading channel at $3-\mathrm{b} / \mathrm{s} / \mathrm{Hz}$ using MRC.

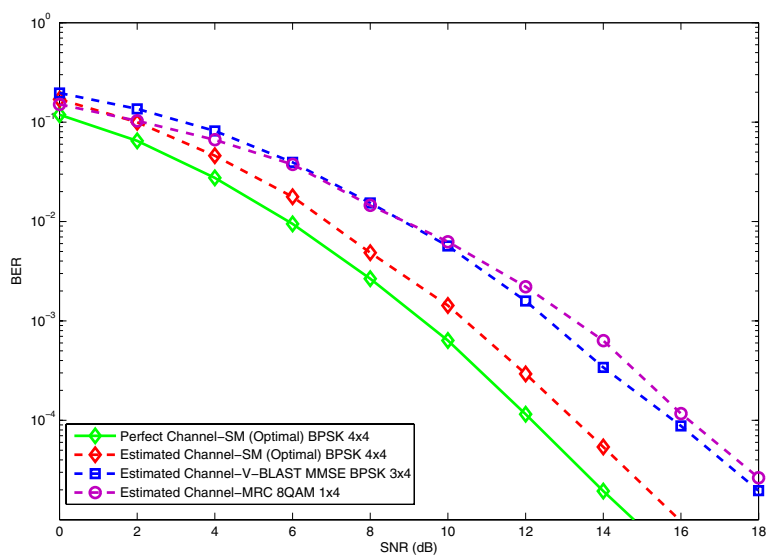

Fig. 6. Performance of the RLS channel estimator for pilot rate $=10 \%$ over a block fading channel at $3-\mathrm{b} / \mathrm{s} / \mathrm{Hz}$ using spatial modulation, V-BLAST, and MRC.

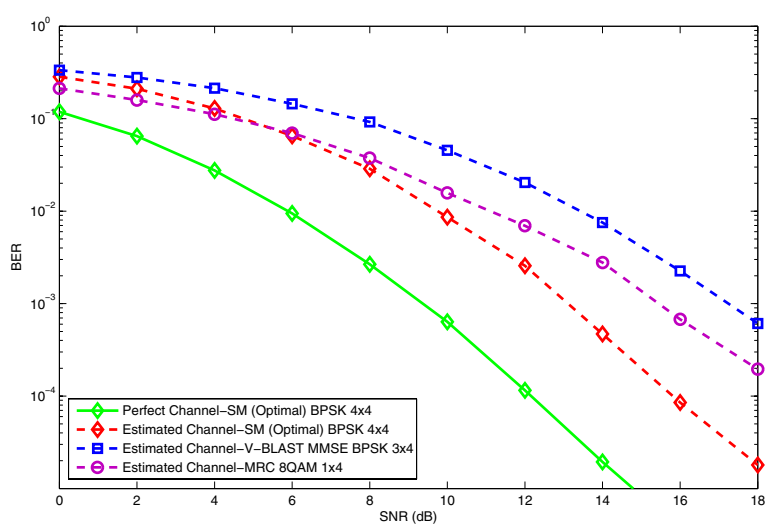

Fig. 7. Performance of the RLS channel estimator for pilot rate $=5 \%$ over a block fading channel at $3-\mathrm{b} / \mathrm{s} / \mathrm{Hz}$ using spatial modulation, V-BLAST, and MRC.

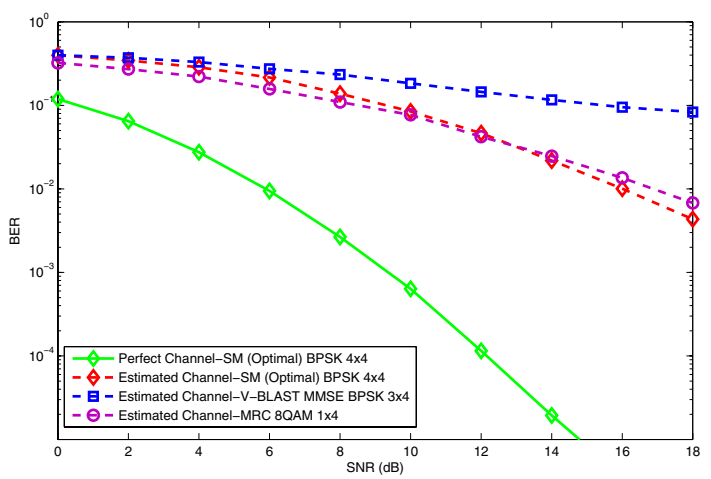

Fig. 8. Performance of the RLS channel estimator for pilot rate $=2 \%$ over a block fading channel at $3-\mathrm{b} / \mathrm{s} / \mathrm{Hz}$ using spatial modulation, V-BLAST, and MRC.

\section{REFERENCES}

[1] H. Haas and S. McLaughlin, eds., "Next Generation Mobile Access Technologies: Implementing TDD," Cambridge, U.K.: Cambridge Univ. Press, Jan. 2008

[2] I. E. Telatar, "Capacity of Multi-Antenna Gaussian Channels," European Trans. Telecommun., vol. 10, no. 6, pp. 558-595, Nov./Dec. 1999.

[3] G. J. Foschini, M. J. Gans, and J. M. Kahn, "Fading Correlation and its Effect on the Capacity of Multielement Antenna Systems," IEEE Trans. Commun., vol. 48, no. 3, pp. 502-513, Mar. 2000.

[4] S. Loyka and G. Tsoulos, "Estimating MIMO System Performance Using the Correlation Matrix Approach," IEEE Commun. Lett., vol. 6, no. 1, pp. 19-21, Jan. 2002.

[5] M. Chiani, M. Z. Win, and A. Zanella, "On the Capacity of Spatially Correlated MIMO Rayleigh-Fading Channels," IEEE Trans. Inf. Theory, vol. 49, no. 10, pp. 2363-2371, Oct. 2003.

[6] S. Catreux, P. F. Driessen, and L. J. Greenstein, "Simulation Results for an Interference-Limited Multiple-Input Multiple-Output Cellular System," IEEE Commun. Lett., vol. 4, no. 11, pp. 334-336, Nov. 2000.

[7] J. Jeganathan, A. Ghrayeb, and L. Szczecinski, "Spatial Modulation: Optimal Detection and Performance Analysis," IEEE Commun. Lett., vol. 12, no. 8, pp. 545-547, Aug. 2008.

[8] R. Y. Mesleh, H. Haas, C. W. Ahn, and S. Yun, "Spatial ModulationA New Low Complexity Spectral Efficiency Enhancing Technique," in Proc. First Int. Conf. Commun. and Networking in China, pp. 1-5, Oct. 2006.

[9] R. Y. Mesleh, H. Haas, S. Sinanović, C. W. Ahn, and S. Yun, "Spatial Modulation,” IEEE Trans. Veh. Technol., vol. 57, no. 4, pp. 2228-2241, July 2008.

[10] A. H. Sayed, "Fundamentals of Adaptive Filtering," New York: Wiley Interscience, 2003.

[11] R. Böhnke, D. Wübben, V. Kühn, and K. D. Kammeyer, "Reduced complexity MMSE detection for BLAST architectures," in Proc. IEEE Global Telecommunications Conference, vol. 4, pp. 2258-2262, Dec. 2003. 